\title{
Peptide ligand and PEG-mediated long-circulating liposome targeted to FGFR overexpressing tumor in vivo
}

\author{
Lulu Cai ${ }^{1,3, \dagger}$ \\ Xianhuo Wang ${ }^{4, \dagger}$ \\ Wenwen Wangl, \\ Neng Qiu' \\ Jiaolin Wen' \\ Xingmei Duan' \\ $\mathrm{Xia} \mathrm{Li}^{\prime}$ \\ Xiang Chen' \\ Li Yang' \\ Zhiyong Qian' \\ Yuquan Wei' \\ Lijuan Chen ${ }^{1,2}$
}

'State Key Laboratory of Biotherapy, West China Hospital, West China Medical School, Sichuan University, ${ }^{2}$ State Key Laboratory Breeding Base of Systematic Research, Development and Utilization of Chinese Medicine, Chengdu University of Traditional Chinese Medicine, Chengdu, Sichuan, ${ }^{3}$ Sichuan Academy of Medical Sciences and Sichuan Provincial People's Hospital, Sichuan Chengdu, China; ${ }^{4}$ Tianjin Medical University Cancer Institute and Hospital, Department of Lymphoma, Sino-US Center for Lymphoma and Leukemia, Key Laboratory of Cancer Prevention and Therapy, Tianjin Medical University, Tianjin, China

tThese authors equally contributed to this research

Correspondence: Lijuan Chen

State Key Laboratory of Biotherapy, West China Hospital, Sichuan University, 4 Keyuan Road, I Gaopeng Street, Chengdu 610041 , Sichuan, People's Republic of China

Tel +8628 85164103

Fax +862885I64060

Email lijuan50I@hotmail.com

Correspondence: Zhiyong Qian State Key Laboratory of Biotherapy, West China Hospital, Sichuan University, 4 Keyuan Road, I Gaopeng Street, Chengdu 61004I, Sichuan, People's Republic of China

Tel +8628 85164103

Fax +862885164060

Email anderson-qian@।63.com
This article was published in the following Dove Press journal:

International Journal of Nanomedicine

13 August 2012

Number of times this article has been viewed

Background and methods: Paclitaxel, a widely used antitumor agent, has limited clinical application due to its hydrophobicity and systemic toxicity. To achieve sustained and targeted delivery of paclitaxel to tumor sites, liposomes composed of egg phosphatidylcholine, cholesterol, and distearolyphosphatidyl ethanolamine-N-poly(ethylene glycol) $\left(\mathrm{PEG}_{2000}\right)$ were prepared by a lipid film method. In addition, the liposomes also contained truncated fibroblast growth factor fragment-PEG-cholesterol as a ligand targeting the tumor marker fibroblast growth factor receptor. Physicochemical characteristics, such as particle size, zeta potential, entrapment efficiency, and release profiles were investigated. Pharmacokinetics and biodistribution were evaluated in C57BL/6 J mice bearing B16 melanoma after intravenous injection of paclitaxel formulated in Cremophor EL (free paclitaxel), conventional liposomes (CL-PTX), or in targeted PEGylated liposomes (TL-PTX).

Results: Compared with CL-PTX and free paclitaxel, TL-PTX prolonged the half-life of paclitaxel by 2.01-fold and 3.40-fold, respectively, in plasma and improved the $\mathrm{AUC}_{0 \rightarrow \mathrm{t}}$ values of paclitaxel by 1.56-fold and 2.31-fold, respectively, in blood. Biodistribution studies showed high accumulation of TL-PTX in tumor tissue and organs containing the mononuclear phagocyte system (liver and spleen), but a considerable decrease in other organs (heart, lung, and kidney) compared with CL-PTX and free paclitaxel.

Conclusion: The truncated fibroblast growth factor fragment-conjugated PEGylated liposome has promising potential as a long-circulating and tumor-targeting carrier system.

Keywords: paclitaxel, truncated fibroblast growth factor fragment, poly(ethylene glycol), liposomes, targeted drug delivery

\section{Introduction}

Paclitaxel is a diterpenoid isolated from Taxus brevifolia, and is approved by the US Food and Drug Administration for the treatment of ovarian and breast cancers. Clinical trials have shown that paclitaxel has antineoplastic activity, particularly against primary ovarian epithelial carcinoma, breast, colon, head and neck cancers, and nonsmall cell lung cancer. Due to its low solubility in water, it is clinically administered dissolved in Cremophor ${ }^{\circledR}$ EL (polyethoxylated castor oil) and ethanol. ${ }^{1,2}$ These formulations are highly allergenic, require extensive premedication, and are responsible for most of the acute toxicity. ${ }^{3-10}$ To overcome these problems, new aqueous-based formulations of paclitaxel that do not require solubilization by Cremophor and ethanol and decrease the systemic toxicities of the drug are currently being developed. ${ }^{11-15}$ Liposomes consist of phospholipids that are highly biocompatible with low toxicity, and they are able to encapsulate both hydrophilic and hydrophobic drugs. Furthermore, one of the strong merits of liposomes is that they can be used for specific purposes, including 
long circulation and targeting, by alteration of their lipid composition or addition of functional agents. ${ }^{16-19}$ During recent decades, encapsulation of chemotherapeutics into liposomal formulations has had reasonable success as a pharmacological strategy in vitro. Sharma et al and Straubinger et al developed a liposomal formulation composed of phosphatidylcholine and phosphatidylglycerol, and showed that the growth-inhibitory activity of paclitaxel-loaded liposomes against a variety of tumor cell lines in vitro was similar to that of the free drug. ${ }^{20-22}$ However, in vivo, their success was limited due to rapid uptake by the reticuloendothelial system, thereby preventing them from reaching the target tissues. ${ }^{20-23}$ The advent of long-circulating liposomes prepared by incorporation of phospholipid-modified polyethyleneglycol (PEG) in the phospholipid bilayer allowed the limitations of conventional liposomes to be overcome and has proven to be a good approach to prolonging the circulation time of the drug and improving its antitumor efficacy. Long-circulating liposomes prepared by surface modification of a flexible hydrophilic polymer such as $\mathrm{PEG}^{24-28}$ are effective as drug carriers for oral, parenteral, and topical administration to avoid unwanted metabolism both in vivo and in vitro. PEGylated liposomal doxorubicin (Doxil $^{\circledR}$, Caelyx ${ }^{\circledR}$; Alza Pharmaceuticals, San Bruno, CA) has shown an ability to inhibit interaction with plasma proteins and mononuclear phagocytes, and can markedly prolong the circulation time, with a terminal half-life of 55 hours in humans. ${ }^{29}$ Sterically stabilized liposomes containing paclitaxel has also been reported. ${ }^{30-33}$

Various tumor cells, such as melanoma, breast cancer, prostate cancer, and nonsmall cell lung cancer are reported to overexpress basic fibroblast growth factor (FGF) and FGF receptors in comparison with normal tissues. ${ }^{34-36}$ In a previous study, we constructed a human truncated basic FGF peptide (30-115 amino acids) containing a basic FGF receptor binding site and part of a heparin-binding site. This truncated basic FGF peptide could effectively bind to FGF receptors on the cell surface, but did not stimulate cell proliferation. ${ }^{37}$ Using this peptide, we have developed FGF receptor-mediated cationic liposomes to target doxorubicin or paclitaxel to tumor cells overexpressing the FGF receptor and to endothelial cells in the tumor neovasculature in vitro and in vivo. Such a delivery system not only improved the uptake of chemotherapeutic agents in tumor tissue and human umbilical vein endothelial cells, but also significantly inhibited tumor growth and improved survival in tumor-bearing mice. ${ }^{38}$ We have also constructed a micellar drug delivery system formulated with cholesterol-block-PEG $\left(\mathrm{COOH}-\mathrm{PEG}_{2000}{ }^{-}\right.$ cholesterol) copolymer and chemically surface-linked it with truncated basic FGF fragments. This micellar system has the advantages of delivering chemotherapeutic agents specifically to tumor cells, and increasing the efficacy of chemotherapeutic agents in vitro. ${ }^{39}$ However, these cationic liposomes and the micellar drug delivery system do not have a noticeably prolonged circulation time in the bloodstream. Therefore, it has been necessary to develop a better tumortargeted drug delivery system with a longer circulation time in the bloodstream.

In this study, liposomes composed of egg phosphatidylcholine, cholesterol, distearolyphosphatidyl ethanolamine-N-poly(ethylene glycol) $\left(\mathrm{DSPE}_{-} \mathrm{PEG}_{2000}\right)$ and COOH-PEG ${ }_{2000}$-cholesterol were prepared using the lipid film method. In addition, truncated basic FGF fragments were conjugated to the distal terminals of PEG previously anchored on liposomes. The purpose of the DSPE-PEG ${ }_{2000}$ and $\mathrm{COOH}-\mathrm{PEG}_{2000}$-cholesterol block copolymer was to keep the liposomes sterically stabilized, after which they were loaded with paclitaxel using the solvent evaporation method. Finally, the truncated basic FGF fragments were conjugated to the carboxyl terminals of $\mathrm{COOH}-\mathrm{PEG}_{2000}$-cholesterol on the surface of the liposomes for targeted delivery to tumor cells. Ideally, such liposomes would substantially increase both passive and active accumulation of paclitaxel in tumor tissue. At the same time, we prepared conventional liposomes containing egg phosphatidylcholine, cholesterol, and paclitaxel (CL-PTX) and also prepared free paclitaxel. The physicochemical and pharmacokinetic properties of the different formulations were then investigated. The suitability of the liposomes as drug carriers to prolong the half-life of paclitaxel was evaluated. In addition, the effect of truncated basic FGF on the tumor-targeting characteristics and biodistribution of the liposomes was elucidated.

\section{Materials and methods Chemicals}

Egg phosphatidylcholine, PEG (molecular weight 2000), cholesterol, Cremophor EL, Dulbecco's modified Eagle's medium, and RPMI 1640 medium were purchased from Sigma (St Louis, MO). Paclitaxel was purchased from Chengdu Mansite Pharmaceutical Co Ltd, Chengdu, China. Acetonitrile (high-performance liquid chromatography, HPLC, grade) was purchased from Fisher Chemical (Loughborough, Leicestershire, UK). Chloroform, methanol (analytical grade), 1-ethyl-3-(3-dimethylaminopropyl)-carbodiimide hydrochloride (EDC), and N-hydroxysuccinimide (NHS), were purchased from Chengdu Kelong Chemical Co Ltd, Chengdu, China. DSPE-PEG 2000 was purchased from Lipoid 
(Ludwigshafen, Germany). COOH-PEG ${ }_{2000}$-cholesterol and truncated basic FGF peptides were prepared and purified in our own laboratory. ${ }^{37,39}$ All other reagents and solvents used were of analytical pure grade.

\section{Animal models}

Male and female C57BL/6 J mice $(\mathrm{n}=90,6-8$ weeks old, 18-22 g) were obtained from the Laboratory Animal Center of Sichuan University in China. All the animals were raised under standard pathogen-free conditions in the Animal Center for Pharmaceutical Research. After a one-week acclimation period, the animals were used for the experiments. Murine B16F10 melanoma cells were purchased from the American Type Culture Collection (Rockville, MD) and cultured in Dulbecco's modified Eagle's medium supplemented with $10 \%(\mathrm{v} / \mathrm{v})$ heat-inactivated phosphate-buffered solution and $10 \mu \mathrm{L} / \mathrm{mL}$ penicillin-streptomycin. The cultures were sustained at $37^{\circ} \mathrm{C}$ in a humidified incubator containing $5 \%$ $\mathrm{CO}_{2}$. The cells were maintained in their exponential growth phase. To prepare tumor-bearing mice, $2 \times 10^{5} \mathrm{~B} 16 \mathrm{~F} 10$ cells in $50 \mu \mathrm{L}$ were subcutaneously injected into the right flank of mice. All of the procedures involved in the animal experiments and the study protocols were approved by our institutional animal care and use committee (Sichuan University, Chengdu, China).

\section{Preparation of paclitaxel liposomes}

The long-circulating paclitaxel-encapsulated liposomes were prepared using a thin film evaporation-extrusion technique. Briefly, paclitaxel, egg phosphatidylcholine, cholesterol, COOH-PEG ${ }_{2000}$-cholesterol, and DSPE-PEG 2000 (2:60:30:5:3 $\mathrm{mol} / \mathrm{mol})$ were dissolved in $4 \mathrm{~mL}$ of methanol and chloroform $(1: 3, \mathrm{v} / \mathrm{v})$ as a mixed solvent at room temperature and dried to a thin film, firstly with nitrogen gas and then under vacuum for several hours. The lipid film was hydrated with $2 \mathrm{~mL}$ of $10 \mathrm{mM}$ 2-(N-morpholino)ethanesulfonic acid (MES) buffer ( $\mathrm{pH} 5.0$ ) at $40^{\circ} \mathrm{C}$ for one hour. To obtain small and homogeneous vesicles, the liposome suspension was sonicated for 10 minutes in a bath-type sonicator (Bransonic 12) followed by three extrusion cycles through polycarbonate filters with $0.2 \mu \mathrm{m}$ pores (Lipex ${ }^{\mathrm{TM}}$ Extruder, Northern Lipids Inc, Vancouver, BC). ${ }^{40}$ For CL-PTX, paclitaxel, egg phosphatidylcholine, and cholesterol (molar ratio, 2:60:30) were dissolved in chloroform/methanol $(3: 1, \mathrm{v} / \mathrm{v})$, and then prepared as for the above description of paclitaxel-loaded targeted PEGylated liposomes (TL-PTX) to obtain uniform CL-PTX. The resulting liposomes were purified on a Sephadex G-75 column to remove the nonencapsulated drug content.

\section{Development of truncated basic FGF-conjugated liposomes}

To activate the terminal carboxyl group of COOH-PEG ${ }_{2000}{ }^{-}$ cholesterol, the paclitaxel-loaded liposomes (with $20 \mathrm{mg} / \mathrm{mL}$ COOH-PEG ${ }_{2000}$-cholesterol in $10 \mathrm{mM}$ MES buffer) were incubated with $400 \mathrm{mM}$ EDC and $100 \mathrm{mM}$ NHS for 15 minutes at room temperature with gentle stirring and then neutralized with $\mathrm{NaOH}(0.01 \%)$ to $\mathrm{pH}$ 7.0. The resulting NHS-activated particles were covalently linked to the $-\mathrm{NH}_{2}$ groups of truncated basic FGF (1\% weight compared with lipid concentration). The reaction was continued at $4{ }^{\circ} \mathrm{C}$ overnight. Unreacted truncated basic FGF, EDC, and NHS were removed by gel filtration. ${ }^{41}$ The final concentration of paclitaxel encapsulated in the liposomes was determined by HPLC assay. The encapsulation efficiency of paclitaxel in CL-PTX and in TL-PTX was calculated according to the following equation:

$$
\mathrm{EE}(\%)=\mathrm{W}_{\text {encapsulated }} / \mathrm{W}_{\text {total }} \times 100 \%
$$

where EE is encapsulation efficiency, $\mathrm{W}_{\text {total }}$ is the total amount of paclitaxel in the liposomes before purification and $\mathrm{W}_{\text {encapsulated }}$ is the amount of paclitaxel encapsulated into the liposomes after purification.

\section{Characterization of paclitaxel- encapsulated liposomes}

Size and zeta-potential determination and atomic force microscopy

The particle size distribution and zeta potential of CL-PTX and TL-PTX were measured by dynamic light scattering (Zetasizer, Nano-ZS, Malvern, UK). At least three different batches were analyzed to achieve a mean value and standard deviation for particle diameter and zeta potential. The shape and structure of TL-PTX were observed by atomic force microscopy. The prepared suspension was diluted with deionized water and deposited onto freshly cleaved mica lamella. The sample was dried for 3 hours at room temperature. Atomic force microscopic images were taken by tapping mode in air on an atomic force microscope (SPA400, Seiko, Tokyo, Japan).

\section{Quantification of liposome-bound peptide}

The amount of liposome-bound peptide was determined using a bicinchoninic acid (BCA) protein assay kit (Pierce, Rockford, IL) following the protocol recommended by the manufacturer. Briefly, triplicates of a truncated basic FGF-modified liposome sample along with duplicates of known serial dilutions of BCA (to build a standard curve), 
were added to a 96-well plate in the same volumes. After mixing samples with $200 \mu \mathrm{L}$ of the BCA working reagent (1:50 ratio of $4 \% \mathrm{CuSO}_{4}$ : $\mathrm{BCA}, \mathrm{Na}_{2} \mathrm{CO}_{3}, \mathrm{NaHCO}_{3}, \mathrm{Na}$ tartrate in $0.1 \mathrm{M} \mathrm{NaOH}$ ), the plate was incubated at $37^{\circ} \mathrm{C}$ for 30 minutes. After cooling to room temperature, absorbance of the purple-colored complex of BCA and $\mathrm{Cu}^{1+}$ (product of protein oxidation by $\mathrm{Cu}^{2+}$ ) was measured at $562 \mathrm{~nm}$ using a Labsystems Multiscan MCC/340 plate reader (Labsystems and Life Sciences International Ltd, London, UK).

\section{In vitro drug release}

Release of the paclitaxel from CL-PTX, TL-PTX, and Cremophor EL in vitro was monitored using a dialysis method. Dialysis was carried out at $37^{\circ} \mathrm{C}$ using Spectra/ Por dialysis membranes with a molecular weight cutoff of $14 \mathrm{kDa}$ and phosphate-buffered saline ( $\mathrm{pH}$ 7.4) as the sink solution. The initial concentration of paclitaxel in the formulations was $2 \mathrm{mg} / \mathrm{mL}$. At scheduled intervals, $1 \mathrm{~mL}$ of the dialysis medium was collected and the same volume of fresh medium was added immediately. The concentration of paclitaxel in dialysis medium was monitored by HPLC assay. Chromatographic separations were carried out on a C18 column $(250 \times 4.6 \mathrm{~mm}, 5 \mu \mathrm{m})$ and samples were eluted using acetonitrile and water $(50: 50, \mathrm{v} / \mathrm{v})$ at a flow rate of $1.0 \mathrm{~mL}$ per minute with the ultraviolet detector at $227 \mathrm{~nm}$. The release percentage was calculated according to the following equation:

$$
\text { Drug release percentage }(\%)=\left(\mathrm{W}_{\text {release }} / \mathrm{W}_{\text {total }}\right) \times 100 \%
$$

where $\mathrm{W}_{\text {total }}$ is the total amount of paclitaxel in the liposomes and $\mathrm{W}_{\text {release }}$ is the amount of paclitaxel released from the liposomes or Cremophor EL into the dialysis medium at any time.

\section{Pharmacokinetics and biodistribution}

The study protocol for the animal experiments complied with the guidelines on animal experimentation for Sichuan University, China. When the B16F10 tumors had reached a volume of about $500 \mathrm{~mm}^{3}$, the C57BL/6 J tumor-bearing mice were randomly and equally divided into three groups, which received free paclitaxel solution, CL-PTX, or TLPTX administered as a single dose of $10 \mathrm{mg}$ paclitaxel $/ \mathrm{kg}$ body weight via the tail vein. Free paclitaxel solution, as the control, was prepared by dissolving $200 \mathrm{mg}$ of paclitaxel in a $10 \mathrm{~mL}$ mixture of Cremophor EL (1.5\%) and ethanol (1.5\%) followed by dilution with $5 \%$ glucose (w/v) to $200 \mathrm{~mL}$ to generate a final concentration of $1 \mathrm{mg} / \mathrm{mL}$. All the free paclitaxel solution mentioned below was made up as the same preparation. The concentration of paclitaxel in the CL-PTX and TL-PTX formulations was also $1 \mathrm{mg} / \mathrm{mL}$.

For the pharmacokinetic studies, the mice were anesthetized by halothane inhalation and bled by eye enucleation at predetermined time points $(0.5,1,3,5,12$, and 24 hours following administration of the experimental treatments). Plasma samples were obtained and centrifuged immediately at $4000 \times \mathrm{g}$ for 10 minutes, and were then stored at $-20^{\circ} \mathrm{C}$ until analysis. For the biodistribution studies, the mice from each group (five mice per point) were sacrificed following collection of blood at predetermined time points. Tissues of interest (tumor, heart, liver, spleen, lung, kidney, and brain) were rapidly excised from each mouse and immediately washed twice with normal saline $(0.9 \% \mathrm{NaCl})$, dried with filter paper, weighed, homogenized, and frozen at $-20^{\circ} \mathrm{C}$ until analysis.

Ultraperformance liquid chromatography (Waters, Milford, MA) was used to determine the paclitaxel concentration in the plasma and tissues. Paclitaxel was extracted by methyl-tertbutyl ether and quantified by reverse-phase ultraperformance liquid chromatography with a $\mathrm{C}_{18}$ column $(2.1 \times 50 \mathrm{~mm}$, $1.7 \mu \mathrm{m}$ ) using an ultraviolet wavelength of $227 \mathrm{~nm}$, with water and acetonitrile (40:60, vol/vol) as the eluant solution. The paclitaxel concentrations were fitted by a Pharmacokinetic Software of Drug and Statistics (edited by the Mathematical Pharmacology Professional Committee of China, version 2.1.1). The data were normalized to tissue weights.

\section{Statistics}

The data were expressed as the mean \pm the standard deviation. Statistical differences were estimated using the Student's $t$-test. The data were considered to be statistically significant at $P<0.05$. Pharmacokinetic parameters were obtained using the practical pharmacokinetic program version 87 (Committee of Mathematic Pharmacology of the Chinese Society of Pharmacology, China). All statistical tests were two-sided, and were performed using SPSS version 16 for Windows statistical software (SPSS Inc, Chicago, IL).

\section{Results}

\section{Physicochemical properties of CL- PTX \pm truncated basic FGF fragments}

Both CL-PTX and TL-PTX were suitable as injectable formulations, and had a small particle size. The shape of the CL-PTX was round or oval, as observed by atomic force microscopy, and is shown in Figure 2C. The mean vesicle diameter of CL-PTX was $137.3 \pm 23.1 \mathrm{~nm}(\mathrm{n}=3)$ which was determined by dynamic light scattering, and the 


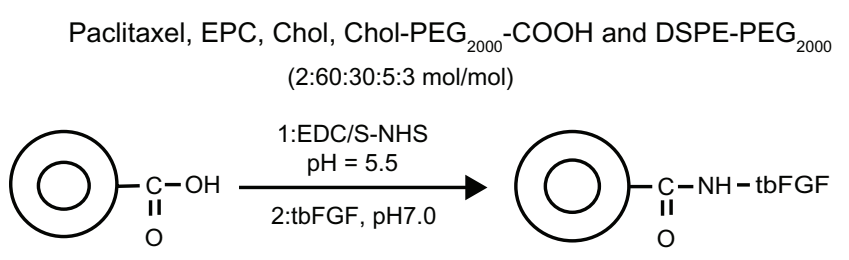

Figure I Synthetic scheme for preparation of targeted PEGylated liposomes. Abbreviations: PEG, poly(ethylene glycol); EPC, egg phosphatidylcholine; Chol, cholesterol; DSPE, distearolyphosphatidyl ethanolamine; EDC, I-ethyl-3-(3dimethylaminopropyl)-carbodiimide hydrochloride; NHS, N-hydroxysuccinimide; tbFGF, truncated basic fibroblast growth factor.

polydispersity index was $0.217 \pm 0.072$ (Table 1 and Figure 2B). The TL-PTX data showed that the mean liposome size increased slightly after attachment of the truncated basic FGF fragment $(142.2 \pm 46.4, \mathrm{n}=3)$, and the polydispersity index was $0.255 \pm 0.086$ (Table 1 and Figure 2A). Zeta potential measurement indicated that the surface charge of CL-PTX and TL-PTX was $-9.20 \pm 0.31 \mathrm{mV}(\mathrm{n}=3)$ and $-5.05 \pm 0.16 \mathrm{mV}(\mathrm{n}=3)$, respectively (Table 1$)$. Because the isoelectric point of the truncated basic FGF fragments was $\mathrm{pH}$ 8.77 , the increase in zeta potential of TL-PTX (compared with CL-PTX, $P<0.05$ ) might have contributed to the positive charge of the truncated basic FGF fragments in the buffer ( $\mathrm{pH}$ 7.4). The average encapsulation efficiency of paclitaxel in CL-PTX and TL-PTX was $89.7 \% \pm 3.6 \%$ and $89.1 \% \pm 2.3 \%$, respectively. The amount of truncated basic FGF conjugated to the liposomal surface was approximately $27.4 \pm 1.8 \mu \mathrm{g}$ truncated basic FGF/mg COOH-PEG ${ }_{2000}$-cholesterol $(\mathrm{n}=3)$, as determined by BCA quantitative protein assay.

\section{Stability of formulated liposomes}

The stability of CL-PTX and TL-PTX was observed over a one-week time course with storage at $4^{\circ} \mathrm{C}$. The results (Table 2) show no significant differences in particle size distribution, zeta potential, and encapsulation efficiency for paclitaxel on any of the days in storage, indicating that CL-PTX and TL-PTX are stable for at least a week when stored at $4^{\circ} \mathrm{C}$. The experiments were performed in triplicate.

\section{Paclitaxel release from TL-PTX in vitro}

The paclitaxel release profile from TL-PTX in vitro is shown in Figure 3. A typical two-phase release profile was observed for TL-PTX, ie, relatively rapid release in the first stage (22.1\%, $0.442 \mathrm{mg}$ versus $2 \mathrm{mg}$ within 8 hours) followed by sustained and slow release over a prolonged period for up to several days. It was found that only $44.1 \%$ ( $0.882 \mathrm{mg}$ versus $2 \mathrm{mg}$ ) was released from the liposomes within 120 hours, while about $88.5 \%$ of the free paclitaxel (1.770 $\mathrm{mg}$ versus $2 \mathrm{mg}$ ) was released into the outside medium. The drug release rate for free paclitaxel was about twice that of TL-PTX. The release profile for CL-PTX was similar to that of TL-PTX.

\section{Pharmacokinetic parameters}

The plasma concentration-time curves and pharmacokinetic parameters after intravenous administration of the different paclitaxel formulations are shown in Figure 4
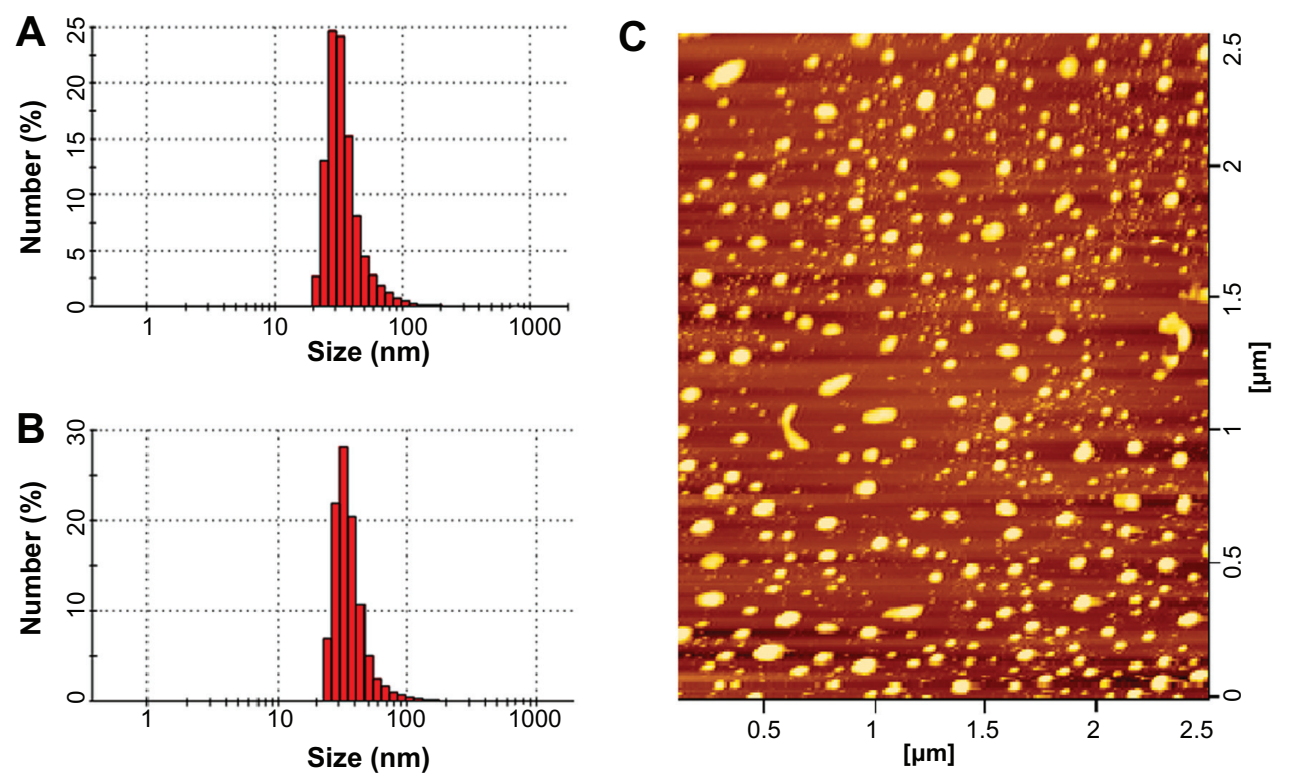

Figure 2 Particle size distribution of paclitaxel-loaded conventional liposomes (A) and paclitaxel-loaded targeted PEGylated liposomes (B), and typical atomic force microscopy image of paclitaxel-loaded targeted PEGylated liposomes (C). 
Table I Particle size and zeta potential (mean \pm SD, $n=3$ ) of CL-PTX and TL-PTX

\begin{tabular}{lllll}
\hline Formulation & Samples & Size $(\mathbf{n m})$ & Polydispersity index & Zeta potential $(\mathbf{m V})$ \\
\hline CL-PTX & $\mathrm{I}$ & 161.2 & 0.286 & -8.94 \\
& 2 & 135.8 & 0.223 & -9.16 \\
& 3 & 115 & 0.143 & -9.55 \\
TL-PTX & Mean \pm SD & $137.3 \pm 23.1$ & $0.217 \pm 0.072$ & $-9.20 \pm 0.31$ \\
& $\mathrm{I}$ & 186.3 & 0.346 & -5.18 \\
& 2 & 146.5 & 0.245 & -5.11 \\
& 3 & 93.8 & 0.175 & -4.87 \\
\hline
\end{tabular}

Abbreviations: CL-PTX, paclitaxel-loaded conventional liposomes; TL-PTX, paclitaxel-loaded targeted PEGylated liposomes; SD, standard deviation.

and Table 3. The highest plasma paclitaxel concentration was observed after injection of the TL-PTX formulation (maximum concentrations of TL-PTX, CL-PTX and free paclitaxel were $18.95 \pm 1.03,7.93 \pm 0.47$, and $5.58 \pm 0.31$, respectively, $P<0.05$ ). This indicates that CL-PTX had a drug concentration-time curve similar to that of the free paclitaxel solution, meaning that paclitaxel was quickly removed from the circulation. In contrast, TL-PTX showed markedly delayed clearance in blood. TL-PTX prolonged the half-life of paclitaxel by 2.01-fold and 3.40-fold in plasma, and improved the $\mathrm{AUC}_{0 \rightarrow \infty}$ values of paclitaxel by 1.56 -fold and 2.31-fold compared with CL-PTX and free paclitaxel, respectively. The paclitaxel in TL-PTX was eliminated rather slowly, with a longer $t_{1 / 2 \beta}(29.12 \pm 1.81$ hours $)$ compared with CL-PTX and free paclitaxel solution. The $t_{1 / 2 \beta}$ of CLPTX (14.48 \pm 1.07 hours) was a little longer than that of free paclitaxel (8.56 \pm 0.91 hours). The $\mathrm{AUC}_{0 \rightarrow \infty}$ of TL-PTX $\left(62.17 \pm 6.92 \mu \mathrm{g} / \mathrm{mL}^{*}\right.$ hour $)$ was significantly larger than that of CL-PTX $(39.88 \pm 2.49 \mu \mathrm{g} / \mathrm{mL} *$ hour$)$ and of free paclitaxel (26.89 $\pm 2.96 \mu \mathrm{g} / \mathrm{mL}^{*}$ hour).

\section{Biodistribution studies}

In our study, the biodistribution of paclitaxel after intravenous injection in the three different preparations were evaluated in a B16 tumor-bearing mouse model. The three preparations consisted of TL-PTX, CL-PTX, and free paclitaxel. The paclitaxel distribution results are presented in Figures 5 and 6 , and show that distribution of paclitaxel in the body could be markedly affected by the different formulations used for encapsulation. The paclitaxel concentration in tumor tissue achieved by TL-PTX was the highest of the three formulations at each time point. TL-PTX increased the concentration of paclitaxel at the tumor site by 1.89 fold $(6.60 \pm 0.57 \mu \mathrm{g} / \mathrm{g}$ versus $3.48 \pm 0.38 \mu \mathrm{g} / \mathrm{g}, P<0.01)$ and 4.66-fold $(6.60 \pm 0.57 \mu \mathrm{g} / \mathrm{g}$ versus $1.41 \pm 0.12 \mu \mathrm{g} / \mathrm{g}$, $P<0.01)$ at 0.5 hours, and 3.06-fold $(0.86 \pm 0.10 \mu \mathrm{g} / \mathrm{g}$ versus $0.28 \pm 0.04 \mu \mathrm{g} / \mathrm{g}, P<0.01)$ and 5.21-fold $(0.86 \pm 0.10 \mu \mathrm{g} / \mathrm{g}$ versus $0.17 \pm 0.03 \mu \mathrm{g} / \mathrm{g}, P<0.01)$ after 24 hours compared with CL-PTX and free paclitaxel, respectively (Table 4). Furthermore, the $\mathrm{AUC}_{0-\infty}$ for TL-PTX in tumors was 4.61fold higher than that for free paclitaxel $(46.43 \pm 8.08 \mathrm{mg} / \mathrm{L} /$ hour versus $10.07 \pm 1.11 \mathrm{mg} / \mathrm{L} /$ hour, $P<0.01)$, 1.81 fold that for CL-PTX $(46.43 \pm 8.08 \mathrm{mg} / \mathrm{L} /$ hour versus $25.77 \pm 0.95 \mathrm{mg} / \mathrm{L} /$ hour, $P<0.01$ ), and the maximum concentration $\left(\mathrm{C}_{\max }\right)$ for TL-PTX in tumor tissue was 5.15fold $(6.60 \pm 0.55 \mathrm{mg} / \mathrm{L}$ versus $1.28 \pm 0.09 \mathrm{mg} / \mathrm{L}, P<0.01)$ higher than that of free paclitaxel and 1.88-fold that of CLPTX $(6.60 \pm 0.55 \mathrm{mg} / \mathrm{L}$ versus $3.51 \pm 0.24 \mathrm{mg} / \mathrm{L}, P<0.01)$. These data suggest that TL-PTX enabled drug accumulation specifically in tumors overexpressing the FGF receptor.

As shown in Figure 6, paclitaxel concentrations from the TL-PTX formulation were significantly $(P<0.05)$ higher in the spleen, but lower $(P<0.05)$ in the lung, heart, and kidney compared with those from the CL-PTX or free paclitaxel

Table 2 Stability of formulated liposomes (mean \pm SD, $n=3$ )

\begin{tabular}{llcccc}
\hline Formulation & Parameters & Day & & \\
\cline { 2 - 5 } & & $\mathbf{0}$ & $\mathbf{I}$ & $\mathbf{3}$ & $\mathbf{7}$ \\
\hline CL-PTX & Size $(\mathrm{nm})$ & $137.3 \pm 23.1$ & $138.1 \pm 24.2$ & $137.5 \pm 23.6$ & $138.1 \pm 25.1$ \\
& Zeta potential $(\mathrm{mV})$ & $9.20 \pm 0.31$ & $9.24 \pm 0.35$ & $9.18 \pm 0.29$ & $9.25 \pm 0.37$ \\
& Encapsulation efficiency $(\%)$ & $89.7 \pm 3.6$ & $89.4 \pm 3.2$ & $88.9 \pm 2.8$ & $89.1 \pm 3.0$ \\
TL-PTX & Size $(\mathrm{nm})$ & $142.3 \pm 46.4$ & $142.6 \pm 46.8$ & $144.1 \pm 47.2$ & $143.6 \pm 46.9$ \\
& Zeta potential $(\mathrm{mV})$ & $5.05 \pm 0.16$ & $5.13 \pm 0.19$ & $4.98 \pm 0.12$ & $5.02 \pm 0.18$ \\
& Encapsulation efficiency $(\%)$ & $89.1 \pm 2.3$ & $89.3 \pm 2.5$ & $88.7 \pm 1.8$ & $88.9 \pm 2.1$ \\
\hline
\end{tabular}

Abbreviations: CL-PTX, paclitaxel-loaded conventional liposomes; TL-PTX, paclitaxel-loaded targeted PEGylated liposomes; SD, standard deviation. 


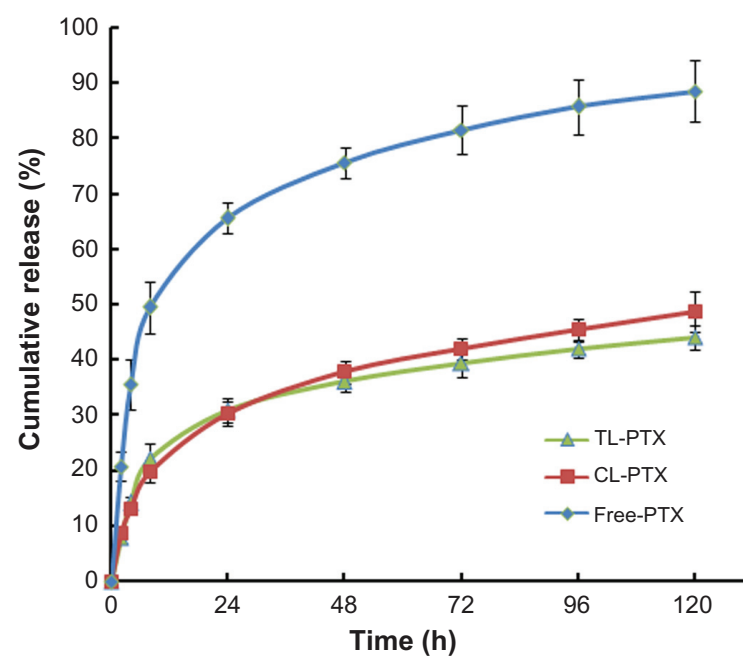

Figure 3 Time course of paclitaxel release from $\operatorname{TL}-\operatorname{PTX}(\mathbf{\Delta}), \mathrm{CL}-\mathrm{PTX}(\mathbf{\square})$, and free $\mathrm{PTX}(\bullet)$ at $37^{\circ} \mathrm{C}$ and $\mathrm{pH}$ 7.0. The paclitaxel Released was separated by dialysis and quantified using high-performance liquid chromatography.

Notes: There were five mice per group per time point. Bars represent the mean \pm standard deviation. Error bars correspond to $95 \%$ confidence intervals.

Abbreviations: CL-PTX, paclitaxel-loaded conventional liposomes; TL-PTX, paclitaxel-loaded targeted PEGylated liposomes; Free PTX, paclitaxel dissolved in Cremophor EL.

formulations. Although the liposomes distributed mainly to the organs of the mononuclear phagocyte system, with most of the accumulation in the liver, paclitaxel concentrations in the liposomal formulation were significantly $(P<0.05)$ lower than those from free paclitaxel in the liver from 0.5 to 5 hours after injection.

\section{Discussion}

In chemotherapy for cancer, the crucial problem is how to avoid the side effects of drugs on healthy tissues. ${ }^{42-47}$ Only

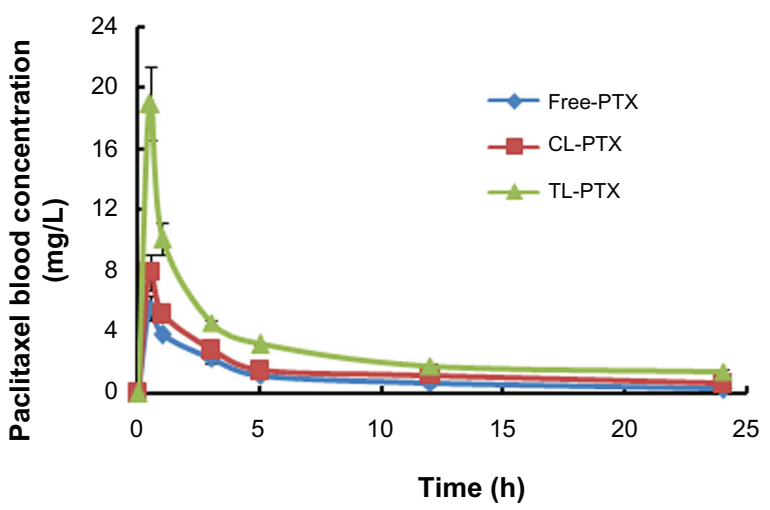

Figure 4 Areas under the curve over 24 hours for paclitaxel in serum after treatment with free PTX $(\downarrow)$, CL-PTX $(\boldsymbol{\square})$, or TL-PTX $(\boldsymbol{\Delta})$.

Notes: There were five mice per group per time point. Bars represent the mean \pm standard deviation.

Abbreviations: CL-PTX, paclitaxel-loaded conventional liposomes; TL-PTX, paclitaxel-loaded targeted PEGylated liposomes; Free PTX, paclitaxel dissolved in Cremophor EL.
$2 \%-5 \%$ of a therapeutic drug dose is taken up by tumor tissue, with more than $90 \%$ taken up by normal tissues. ${ }^{43,46}$ As a result, side effects often result in dose reduction, frequent treatment, or discontinuation of therapy. Recent research in this area has focused on receptor-mediated drug delivery systems based on liposomes. Active targeting of tumor tissue by liposomes can be achieved by modifying the liposome with addition of a targeting ligand, such as an antibody and a peptide. ${ }^{48-51}$ However, the problem with these systems is that the majority of the liposomes are still cleared by the reticuloendothelial system, resulting in short half-lives, low accumulation in tumor tissue, and unwanted deposition in the liver and spleen. To achieve a specific therapeutic effect, both a prolonged blood circulation time and an active tumortargeting effect are essential for an ideal drug delivery system. In our study, a novel formulation was designed for prolonged systemic circulation and selective targeting of tumor tissue showing amplified expression of the FGF receptor.

In this research, the particle size and zeta potential of CL-PTX and TL-PTX formulations were described. The liposomes were observed by atomic force microscopy to characterize their structures further. The particle size of TL-PTX (142.2 $\pm 46.4 \mathrm{~nm})$ was slightly larger than that of CL-PTX $(137.3 \pm 23.1 \mathrm{~nm})$ due to the fact that truncated basic FGF was attached to the surface of the liposomes so as to increase the diameter of TL-PTX. In contrast, part of the negative charge of TL-PTX was neutralized by truncated basic FGF so that the zeta potential of TL-PTX $(-5.05 \pm 0.16 \mathrm{mV})$ was slightly lower than that of CL-PTX $(-9.20 \pm 0.3 \mathrm{mV})$. There is some evidence that neutral or negatively charged liposomes could have a longer circulation time in the bloodstream because of their weaker interaction with serum proteins compared with positively charged liposomes. ${ }^{52}$ An early study using liposomes demonstrated that small neutral unilamellar vesicles $(\leq 100 \mathrm{~nm})$ made from equimolar amounts of saturated phospholipids and cholesterol had a longer circulation time in rats (half-lives up to 20 hours) compared with their anionic counterparts (half-lives less than one hour). ${ }^{53}$ These observations are in agreement with the process of complement opsonization of liposomes, whereby neutral vesicles were poor activators of the complement system when compared with positive or anionic liposomes. ${ }^{54-56}$ Therefore, small-sized neutral vesicles were not efficiently coated with the opsonizing complement proteins, so that they were poorly recognized by Kupffer cells. Truncated basic FGF fragments contain many basic amino residues, which might result in a positive charge on the liposomal surface. The positive charge might induce rapid 
Table 3 Pharmacokinetic parameters for paclitaxel in plasma after intravenous administration of free PTX, CL-PTX, and TL-PTX (mean \pm standard deviation)

\begin{tabular}{|c|c|c|c|c|}
\hline Parameters & Units & Free-PTX & CL-PTX & TL-PTX \\
\hline $\mathrm{T}_{1 / 2}$ & $\mathrm{~h}$ & $8.562 \pm 0.11$ & $14.476 \pm 0.07$ & $29.122 \pm 0.11$ \\
\hline $\mathrm{T}_{\max }$ & $\mathrm{h}$ & $0.5 \pm 0.00$ & $0.5 \pm 0.00$ & $0.5 \pm 0.00$ \\
\hline$C_{\max }$ & $\mathrm{mg} \mathrm{L}^{-1}$ & $5.58 \pm 0.31$ & $7.93 \pm 0.47$ & $18.95 \pm 1.03 *$ \\
\hline$A \cup C_{0>t}$ & $m g L^{-1} h$ & $26.9 \pm 1.97$ & $39.9 \pm 2.67$ & $62.17 \pm 4.72^{*}$ \\
\hline $\mathrm{AUC}_{0 \rightarrow \infty}$ & $m g L^{-1} h$ & $30.01 \pm 2.96$ & $52.98 \pm 2.49$ & $120.00 \pm 6.92 *$ \\
\hline $\mathrm{AUMC}_{0 \rightarrow \infty}$ & & $160.89 \pm 7.15$ & $281.93 \pm 12.29$ & $480.57 \pm 16.92 *$ \\
\hline $\mathrm{MRT}_{0 \rightarrow \infty}$ & $\mathrm{h}$ & $9.15 \pm 0.27$ & $16.42 \pm 0.36$ & $3.50 \pm 0.93$ \\
\hline$v_{d}$ & $\mathrm{~L} \mathrm{~kg}^{-1}$ & $4.12 \pm 0.04$ & $3.94 \pm 0.08$ & $3.50 \pm 0.02$ \\
\hline$C L$ & $\mathrm{~L} \mathrm{~h}^{-1} \mathrm{~kg}^{-1}$ & $0.33 \pm 0.03$ & $0.19 \pm 0.04$ & $0.08 \pm 0.06 *$ \\
\hline
\end{tabular}

Notes: Each data was from five mice. ${ }^{*} P$ values $\leq 0.05$ were considered to be statistically significant compared with the free PTX group.

Abbreviations: CL-PTX, paclitaxel-loaded conventional liposomes; TL-PTX, paclitaxel-loaded targeted PEGylated liposomes; Free PTX, paclitaxel dissolved in Cremophor EL; $\mathrm{T}_{\max }$, time to maximum plasma concentration of drug; $\mathrm{C}_{\max }$, maximum plasma concentration of drug; $\mathrm{T}_{1 / 2}$, elimination half life; $\mathrm{CL}$, clearance; $\mathrm{MRT}$, mean residence time; $\mathrm{V}_{\mathrm{d}}$, apparent volume of distribution; $\mathrm{AUC}_{0 \rightarrow \infty}$, area under the plasma concentration-time curve.

uptake by the reticuloendothelial system and nonspecific binding with unintended cells, such as erythrocytes, after intravenous administration. To overcome this problem, we chose egg phosphatidylcholine, cholesterol, DSPE-PEG ${ }_{2000}$, and $\mathrm{COOH}-\mathrm{PEG}_{2000}$-cholesterol as the primary elements of TL-PTX to achieve a surface charge of $-5.05 \pm 0.16 \mathrm{mV}$. The surface charge was close to neutral, and enabled paclitaxel to have prolonged circulation time in the bloodstream and improved its penetration and accumulation at tumor sites. Further, the PEG chains in COOH-PEG 2000 -cholesterol and DSPE-PEG ${ }_{2000}$ were expected to limit interaction with the reticuloendothelial system and other nontarget cells. The pharmacokinetics and biodistribution results for TL-PTX in vivo confirmed this hypothesis.

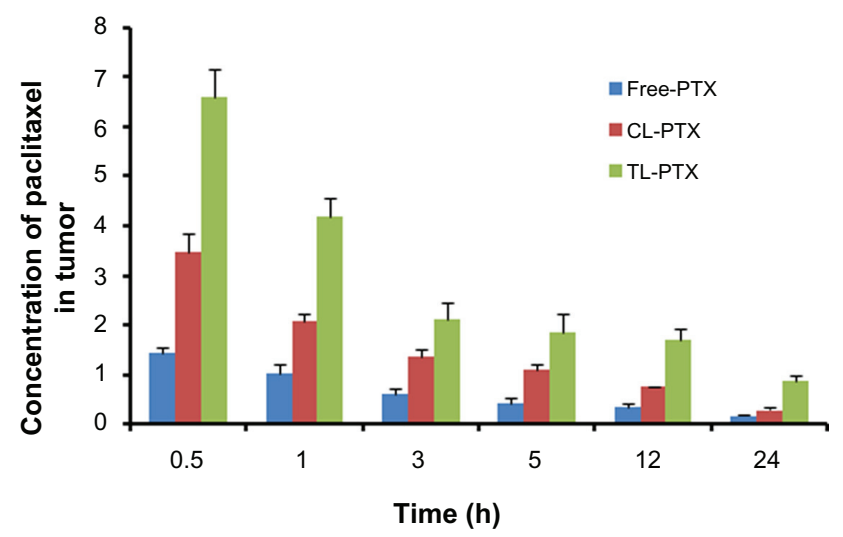

Figure 5 Mean paclitaxel concentration in tumor tissue at each time point after treatment with free PTX, CL-PTX, and TL-PTX. Paclitaxel was extracted from the tumors and measured by ultraperformance liquid chromatography.

Notes: There were five mice per group per time point. Bars represent the mean \pm standard deviation.

Abbreviations: CL-PTX, paclitaxel-loaded conventional liposomes; TL-PTX, paclitaxel-loaded targeted PEGylated liposomes; Free PTX, paclitaxel dissolved in Cremophor EL.
In vitro release of paclitaxel from the free paclitaxel, CL-PTX, and TL-PTX formulations was studied at $37^{\circ} \mathrm{C}$ in buffer ( $\mathrm{pH}$ 7.4). The initial concentration of paclitaxel was selected as $2 \mathrm{mg} / \mathrm{mL}$ to enable a series of drug concentrations in dialysis medium to be appropriately detected after release from the three formulations. Relatively rapid release from free paclitaxel and slow release from CL-PTX and TL-PTX was observed. It was demonstrated that paclitaxel could pass through the dialysis membrane entirely into the dissolution medium and that paclitaxel could be released slowly from the liposomes. Therefore, CL-PTX and TL-PTX showed greatly increased stability compared with free paclitaxel.

Because the concentration of paclitaxel in the extracellular fluid $\left(\mathrm{pH} 7.4,37^{\circ} \mathrm{C}\right)$ was relatively low, paclitaxel was released rapidly over $0-12$ hours from TL-PTX and CLPTX as a result of being driven by a concentration gradient. After 12 hours, the drug concentration in extracellular fluid reached a certain level and the effect of the concentration gradient became smaller, enabling slower release of paclitaxel from TL-PTX and CL-PTX, which ensured a long-circulating effect. This attenuated drug release might result in a more favorable pharmacokinetic profile in vivo. ${ }^{57-59}$

To evaluate the pharmacokinetics and tissue distribution of PEG-mediated and truncated basic FGF-mediated paclitaxel liposomes, we used C57BL/6J tumor-bearing mice and selected $10 \mathrm{mg}$ paclitaxel/ $\mathrm{kg}$ body weight as a single dose to be given via the tail vein consistent with a previous report. ${ }^{60}$ Our present work provided an overall profile for circulation of the drug in the bloodstream, the main body tissues, and the tumor tissue. The pharmacokinetic parameters for paclitaxel changed significantly and were observed for TL-PTX, CL-PTX, and free paclitaxel. The higher plasma concentration of TL-PTX and CL-PTX compared with free paclitaxel in mice might 
A

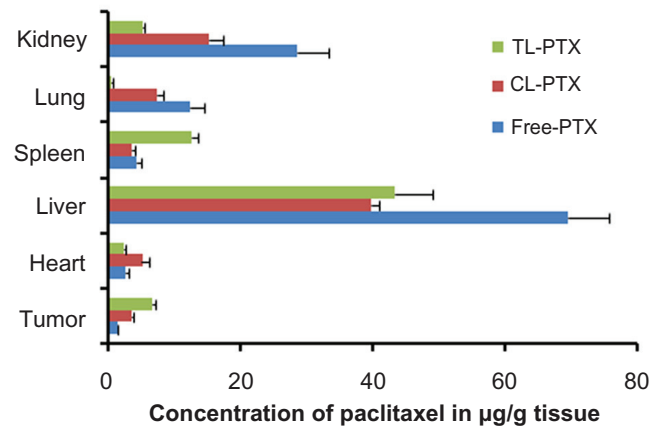

C

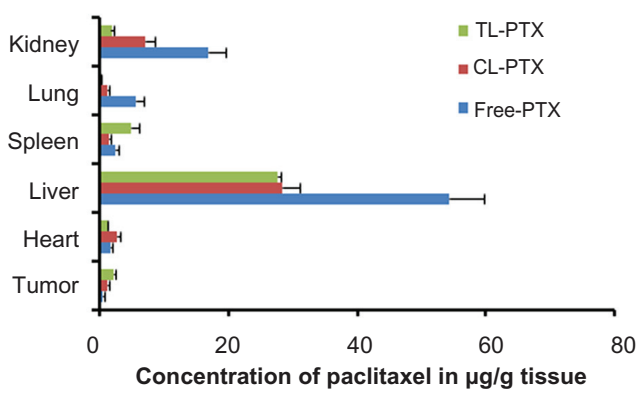

E

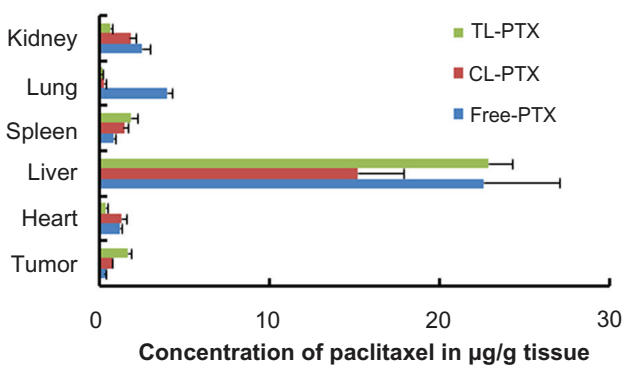

B

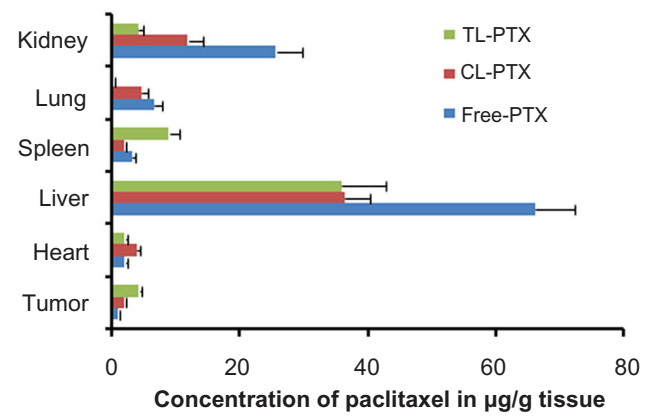

D

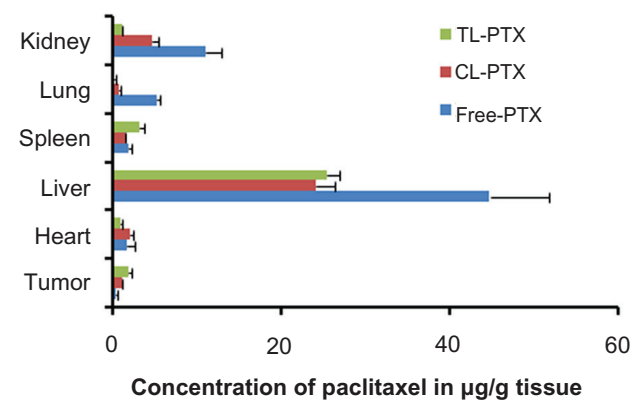

$\mathbf{F}$

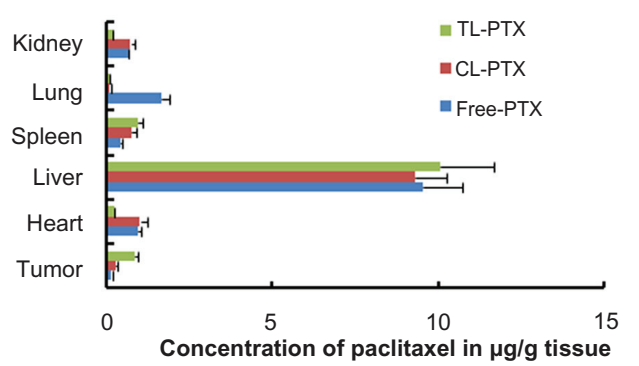

Figure 6 Mean paclitaxel concentration in tumor, lungs, heart, spleen, liver, and kidneys of mice treated with free PTX, CL-PTX, and TL-PTX. Mice were sacrificed at the indicated time points of (A) 0.5, (B) I, (C) 3, (D) 5, (E) 12, and (F) 24 hours after administration, and paclitaxel was extracted from the tissues and measured by ultraperformance liquid chromatography.

Notes: There were five mice per group per time point. Bars represent the mean \pm standard deviation.

Abbreviations: CL-PTX, paclitaxel-loaded conventional liposomes; TL-PTX, paclitaxel-loaded targeted PEGylated liposomes; Free PTX, paclitaxel dissolved in Cremophor EL.

be due to slower release of paclitaxel from the liposomes, such that paclitaxel in a liposomal form could circulate in the bloodstream for the required period of time. In addition, the slow clearance of paclitaxel from TL-PTX compared with CL-PTX might be due to the relatively better stability

Table 4 Increase versus time of distribution of TL-PTX in the tumors compared with CL-PTX and free PTX, respectively

\begin{tabular}{llllll}
\hline Folds vs time & $\mathbf{0 . 5} \mathbf{h}$ & $\mathbf{I} \mathbf{~}$ & $\mathbf{3} \mathbf{~ h}$ & $\mathbf{5} \mathbf{~ h}$ & $\mathbf{1 2} \mathbf{~}$ \\
\hline CL-PTX & 1.89 & 2.04 & 1.69 & 1.58 & 2.29 \\
Free-PTX & 4.66 & 4.48 & 3.61 & 4.40 & 5.04 \\
\hline
\end{tabular}

Abbreviations: CL-PTX, paclitaxel-loaded conventional liposomes; TL-PTX, paclitaxel-loaded targeted PEGylated liposomes; Free PTX, paclitaxel dissolved in Cremophor EL. of TL-PTX in vivo. The reasons for its better stability might be associated with the physical barrier properties of the PEG coating, which might slow drug release from the liposomes and limit interaction with components of plasma. The slower biodegradability of TL-PTX in the bloodstream could enhance accumulation of paclitaxel in solid tumor vasculature.

Administration of TL-PTX and CL-PTX altered the distribution of paclitaxel in the tissues, with higher uptake in the tumor and spleen and lower uptake in other tissues compared with free paclitaxel. Importantly, TL-PTX showed significantly greater accumulation than CL-PTX and free paclitaxel in tumor tissue. The underlying mechanisms might be two-fold. First, long-circulating liposomes 
may slowly accumulate in tumor tissue and protect the encapsulated agent from enzymatic degradation, ultimately reaching high levels in tumor tissue due to the enhanced permeability and retention effect. Second, the truncated basic FGF might specifically target tumor cells by binding with FGF receptors, resulting in specific uptake of paclitaxel.

The lower concentration of paclitaxel released from TL-PTX than from CL-PTX and free paclitaxel in the lungs, heart, and kidneys at all time points might be due to the specific tumor targeting function of truncated basic FGF-modified liposomes, suggesting attenuated toxicity in healthy tissues.

During the first 5 hours following administration, liposomes distributed mainly to the bloodstream, tumor, and spleen. The concentration of free paclitaxel in the liver was higher than that of TL-PTX or CL-PTX, indicating that these liposomal formulations had a tendency to escape from phagocytic cells in the blood because of their small particle size and PEG coating over a certain time period. However, TL-PTX and CL-PTX accumulated in the liver to the same extent as free paclitaxel after 24 hours, suggesting that the liposomal formulations were still recognized as foreign material and cleared by phagocytic cells of the mononuclear phagocyte system, which are abundant in certain organs, such as the liver and spleen. Nevertheless, the higher level of paclitaxel released from TL-PTX reaching the blood and tumor tissue suggests that TL-PTX achieved targeting and had long-circulating effects.

The level of TL-PTX in the bloodstream was higher than for CL-PTX or free paclitaxel during the 5 hours following injection, possibly for two reasons, ie, TL-PTX might slow drug release from the liposomes and limit interaction with plasma components, and clearance of the drug from TL-PTX was slower. The slower biodegradability of TL-PTX did not enhance drug accumulation in the organs of the mononuclear phagocyte system, but did improve accumulation in the solid tumor vasculature. The results of our study also demonstrate that the additional benefit of less accumulation of TL-PTX in the organs of the mononuclear phagocyte system compared with CL-PTX and free PTX diminished over time, ie, at 12 hours and 24 hours after injection. At the same time, TLPTX did not decrease accumulation of paclitaxel in tumor tissue. The higher paclitaxel concentration in the bloodstream and tumor tissue noted in the group treated with TL-PTX suggests that TL-PTX had targeted and long-circulating effects, which might enhance the therapeutic efficacy and reduce the systemic toxicity of antitumor drugs.
TL-PTX was targeted and long-circulating, which could maintain the concentration of the drug in the bloodstream and control any burst effect. It could also reduce the concentration of the drug in normal tissues, thereby reducing its toxicity. In this study, only paclitaxel was selected as the model drug. The main dose-limiting toxicity of paclitaxel was neutropenia, and TL-PTX accumulated in the bloodstream for a long time. Paclitaxel may not be very suitable for this new drug delivery system, but this does not mean that this new system cannot be used for other chemotherapeutic drugs. Furthermore, although TL-PTX accumulated to a higher degree than CL-PTX and free paclitaxel in the spleen, the paclitaxel concentration in the spleen was very low compared with levels in other organs. In other words, a small amount of paclitaxel in the spleen did not lead to important toxicity. In contrast, the concentration of free paclitaxel was relatively high in the heart, kidney and lung, which are the organs mainly at risk of toxicity, suggesting that the TL-PTX was safer compared to the free paclitaxel.

\section{Conclusion}

In conclusion, PEG-mediated and truncated basic FGFmediated liposomes were developed in this study, with a prolonged circulation time in the bloodstream and enhanced accumulation of paclitaxel, a lipophilic anticancer drug, in tumor tissues. Longer persistence of the liposomes in the blood enabled better targeting of circulating tumor cells. These benefits, in turn, might improve the therapeutic efficacy of paclitaxel, enabling more specific tumor therapy.

\section{Acknowledgments}

This work was supported by the National Key Technologies R\&D Program (2009ZX09501-015), the Natural Science Foundation of China (81071251), and the Open Research Fund of State Key Laboratory Breeding Base of Systematic Research, Development, and Utilization of Chinese Medicine. We acknowledge Minghai Tang for her help in the pharmacokinetic and biodistribution studies.

\section{Disclosure}

The authors report no conflicts of interest in this work.

\section{References}

1. Chervinsky D, Brecher M, Hoelcle M. Cremophor-EL enhances Taxol efficacy in a multi-drug resistant C1300 neuroblastoma cell line. Anticancer Res. 1993;13:93-96.

2. Webster L, Linsenmeyer M, Millward M, Morton C, Bishop J, Woodcock D. Measurement of Cremophor EL following Taxol: plasma levels sufficient to reverse drug exclusion mediated by the multidrug-resistant phenotype. J Natl Cancer Inst. 1993;85:1685-1690. 
3. Fjällskog ML, Frii L, Bergh J. Is Cremophor EL, solvent for paclitaxel, cytotoxic? Lancet. 1993;342:873.

4. Rowinsky E, Eisenhauer E, Chaudhry V, Arbuck S, Donehower R. Clinical toxicities encountered with paclitaxel (Taxol). Semin Oncol. 1993;20(4 Suppl 3):1-15.

5. Dorr RT. Pharmacology and toxicology of Cremophor EL diluent. Ann Pharmacother. 1994;28(5):S11-S14.

6. Goldspiel BR. Pharmaceutical issues: preparation, administration, stability, and compatibility with other medications. Ann Pharmacother. 1994;28:23-26.

7. Song D, Hsu LF, Au JLS. Binding of Taxol to plastic and glass containers and protein under in vitro conditions. J Pharm Sci. 1996;85: 29-31.

8. Kongshaug M, Cheng LS, Moan J, Rimington C. Interaction of Cremophor EL with human plasma. Int J Biochem. 1991;23:473-478.

9. Sykes E, Woodburn K, Decker D, Kessel D. Effects of Cremophor EL on distribution of Taxol to serum lipoproteins. Br J Cancer. 1994;70: 401-404.

10. Gao JF, Guan S, Deng WJ, et al. Clinical study of comparing LP and TP regimens in the treatment of advanced non-small cell lung cancer. Chinese-German J Clin Oncol. 2010;9:198-200.

11. Zhang Q, Huang XE, Gao LL. A clinical study on the premedication of paclitaxel liposome in the treatment of solid tumors. Biomed Pharmacother. 2009;63:603-607.

12. Holvoet C, Vander HY, Lories G, et al. Preparation and evaluation of paclitaxel-containing liposomes. Pharmazie. 2007;62:126-132.

13. Yang T, Cui FD, Chai MK, et al. Liposome formulation of paclitaxel with enhanced solubility and stability. Drug Deliv. 2007;14: 301-308.

14. Stinchcombe TE. Nanoparticle albumin-bound paclitaxel: a novel Cremophor EL-free formulation of paclitaxel. Nanomedicine (Lond). 2007;2:415-423.

15. Hureaux J, Lagarce F, Gagnadoux F, et al. Lipid nanocapsules: readyto-use nanovectors for the aerosol delivery of paclitaxel. Eur J Pharm Biopharm. 2009;73:239-246.

16. Yang T, Choi MK, Cui FD, et al. Antitumor effect of paclitaxel-loaded PEGylated immunoliposomes against human breast cancer cells. Pharm Res. 2007;24:2402-2411.

17. Weiner AL. Liposomes as carriers for polypeptides. Adv Drug Deliv Rev. 1989;3:307-341.

18. Zhao PQ, Wang HJ, Yu M, et al. Paclitaxel-loaded, folic-acid-targeted and TAT-peptide-conjugated polymeric liposomes: in vitro and in vivo evaluation. Pharm Res. 2010;27:1914-1926.

19. Meng SY, Su B, Li W, et al. Integrin-targeted paclitaxel nanoliposomes for tumor therapy. Med Oncol. 2011;28:1180-1187.

20. Sharma A, Mayhew E, Straubinger RM. Antitumor effect of Taxolcontaining liposomes in a Taxol-resistant murine tumor model. Cancer Res. 1993;53:5877-5881.

21. Balasubramanian SV, Straubinger RM. Taxol-lipid interactions: Taxoldependent effects on the physical properties of model membranes Biochemistry. 1994;33:8941-8947.

22. Sharma A, Sharma US, Straubinger RM. Paclitaxel-liposomes for intracavitary therapy of intraperitoneal P388 leukemia. Cancer Lett. 1996;107:265-272.

23. Lin YB, Xie R, Lin YZ. Therapeutic effect of paclitaxel liposome combined with cisplatin for III-IV degrees of cervical cancer. Chin J Woman Child Health Res (Chinese). 2008;19:571-573.

24. Immordino ML, Dosio F, Cattel L. Stealth liposomes: review of the basic science, rationale, and clinical applications, existing and potential. Int J Nanomedicine. 2006;1:297-315.

25. Torchilin VP, Trubetskoy VS. Which polymers can make nanoparticulate drug carriers long-circulating? Adv Drug Deliv Rev. 1995;16: 141-155.

26. Klibanov AL, Maruyama K, Torchilin VP, Huang L. Amphipathic polyethylene glycols effectively prolong the circulation time of liposomes. FEBS Lett. 1990;268:235-237.

27. Blume G, Cevc G. Molecular mechanism of the lipid vesicle longevity in vivo. Biochim Biophys Acta. 1993;1146:157-168.
28. Senior J, Delgado C, Fisher D, Tilcock C, Gregoriadis G. Influence of surface hydrophilicity of liposomes on their interaction with plasma protein and clearance from the circulation: studies with poly (ethylene glycol)-coated vesicles. Biochim Biophys Acta. 1991;1062:77-82.

29. Park J. Liposome-based drug delivery in breast cancer treatment. Breast Cancer Res. 2002;4:95-99.

30. Yang T, Cui FD, Choi MK, et al. Enhanced solubility and stability of PEGylated liposomal paclitaxel: in vitro and in vivo evaluation. Int $J$ Pharm. 2007;338:317-326.

31. Yoshizawa Y, Kono Y, Ogawara K, et al. PEG liposomalization of paclitaxel improved its in vivo disposition and anti-tumor efficacy. Int J Pharm. 2011;412:132-141.

32. Crosasso P, Ceruti M, Brusa P, Arpicco S, Dosio F, Cattel L. Preparation, characterization and properties of sterically stabilized paclitaxelcontaining liposomes. J Control Release. 2000;63: 19-30.

33. Huang Y, Chen XM, Xiang B, et al. Antiangiogenic activity of sterically stabilized liposomes containing paclitaxel (SSL-PTX): in vitro and in vivo. AAPS Pharm Sci Tech. 2010;11:752-759.

34. Penault-Llorca F, Bertucci F, Adélaïde J, et al. Expression of FGF and FGF receptor genes in human breast cancer. Int $J$ Cancer. 1995;61:170-176.

35. Danielsen T, Rofstad EK. VEGF, bFGF and EGF in the angiogenesis of human melanoma xenografts. Int J Cancer. 1998;76:836-841.

36. Kwabi-Addo B, Ozen M, Ittmann M. The role of fibroblast growth factors and their receptors in prostate cancer. Endocr Relat Cancer. 2004; 11:709-724.

37. He Q, Wei Y, Tian L, et al. Inhibition of tumor growth with a vaccine based on xenogeneic homologous fibroblast growth factor receptor-1 in mice. J Biol Chem. 2003;278:21831-21836.

38. Chen X, Wang X, Wang Y, et al. Improved tumor-targeting drug delivery and therapeutic efficacy by cationic liposome modified with truncated bFGF peptide. J Control Release. 2010;145:17-25.

39. Cai L, Qiu N, Li X, et al. A novel truncated basic fibroblast growth factor fragment-conjugated poly (ethylene glycol)-cholesterol amphiphilic polymeric drug delivery system for targeting to the FGFRoverexpressing tumor cells. Int J Pharm. 2011;408:173-182.

40. Sharma A, Mayhew E, Bolcsak L, et al. Activity of paclitaxel liposome formulations against human ovarian tumor xenografts. Int J Cancer. 1997;71:103-107.

41. Savva M, Duda E, Huang L. A genetically modified recombinant tumor necrosis factor- $\alpha$ conjugated to the distal terminals of liposomal surface grafted polyethyleneglycol chains. Int J Pharm. 1999;184: $45-51$.

42. Redd WH, Montgomery GH, DuHamel KN. Behavioral intervention for cancer treatment side effects. J Natl Cancer Inst. 2001;93:810-823.

43. Cheong I, Huang X, Bettegowda C, et al. A bacterial protein enhances the release and efficacy of liposomal cancer drugs. Science. 2006;314: $1308-1311$.

44. Lotfi-Jam K, Carey M, Jefford M, Schofield P, Charleson C, Aranda S. Nonpharmacologic strategies for managing common chemotherapy adverse effects: a systematic review. J Clin Oncol. 2008;26: 5618-5629.

45. Dharap S, Wang Y, Chandna P, et al. Tumor-specific targeting of an anticancer drug delivery system by LHRH peptide. Proc Natl Acad Sci USA. 2005;102:12962-12967.

46. Moghimi SM, Hunter AC, Murray JC. Nanomedicine: current status and future prospects. FASEB J. 2005;19:311-330.

47. Suo T, Ge W, Zhang JZ, et al. 17 cases of advanced non-small cell lung cancer treated with paclitaxel liposome plus nedaplatin. ChineseGerman Journal of Clinical Oncology. 2012;11:196-198.

48. Torchilin VP, Levchenko TS, Rammohan R, Papahadjopoulos-Sternberg B, D'Souza GG. Cell transfection in vitro and in vivo with nontoxic TAT peptide-liposome-DNA complexes. Proc Natl Acad Sci U S A. 2003;100:1972-1977.

49. Yu W, Pirollo KF, Yu B, et al. Enhanced transfection efficiency of a systemically delivered tumor-targeting immunolipoplex by inclusion of a pH-sensitive histidylated oligolysine peptide. Nucleic Acids Res. 2004;32(5):e48. 
50. Kawakami K, Kawakami M, Puri RK. IL-13 receptor-targeted cytotoxin cancer therapy leads to complete eradication of tumors with the aid of phagocytic cells in nude mice model of human cancer. $J$ Immunol. 2002;169:7119-7126.

51. Nakase M, Inui M, Okumura K, Kamei T, Nakamura S, Tagawa T. p53 gene therapy of human osteosarcoma using a transferrin-modified cationic liposome. Mol Cancer Ther. 2005;4:625-631.

52. Moghimi SM, Hunter AC, Murray JC. Long-circulating and targetspecific nanoparticles: theory to practice. Pharmacol Rev. 2001;53: 283-318.

53. Senior J, Gregoriadis G. Is half-life of circulating liposomes determined by changes in their permeability? FEBS Lett. 1982;145:109-114.

54. Volanakis JE, Wirtz KWA. Interaction of C-reactive protein with artificial phosphatidylcholine bilayers. Nature. 1979;281:155-157.

55. Chonn A, Cullis P, Devine D. The role of surface charge in the activation of the classical and alternative pathways of complement by liposomes. J Immunol. 1991;146:4234-4241.
56. Devine DV, Bradley AJ. The complement system in liposome clearance: can complement deposition be inhibited? Adv Drug Deliv Rev. 1998; 32:19-29.

57. Lundberg B, Risovic V, Ramaswamy M, Wasan K. A lipophilic paclitaxel derivative incorporated in a lipid emulsion for parenteral administration. J Control Release. 2003;86:93-100.

58. Gabizon A, Horowitz AT, Goren D, et al. Targeting folate receptor with folate linked to extremities of poly (ethylene glycol)-grafted liposomes: in vitro studies. Bioconjug Chem. 1999;10:289-298.

59. Lee RJ, Low PS. Folate-mediated tumor cell targeting of liposome-entrapped doxorubicin in vitro. Biochim Biophys Acta. 1995; 1233:134-144.

60. Gustafson DL, Merz AL, Long ME. Pharmacokinetics of combined doxorubicin and paclitaxel in mice. Cancer Lett. 2005;220:161-169.
International Journal of Nanomedicine

\section{Publish your work in this journal}

The International Journal of Nanomedicine is an international, peerreviewed journal focusing on the application of nanotechnology in diagnostics, therapeutics, and drug delivery systems throughout the biomedical field. This journal is indexed on PubMed Central, MedLine, CAS, SciSearch $\AA$, Current Contents ${ }^{\circledR} /$ Clinical Medicine,

\section{Dovepress}

Journal Citation Reports/Science Edition, EMBase, Scopus and the Elsevier Bibliographic databases. The manuscript management system is completely online and includes a very quick and fair peer-review system, which is all easy to use. Visit http://www.dovepress.com/ testimonials.php to read real quotes from published authors. 